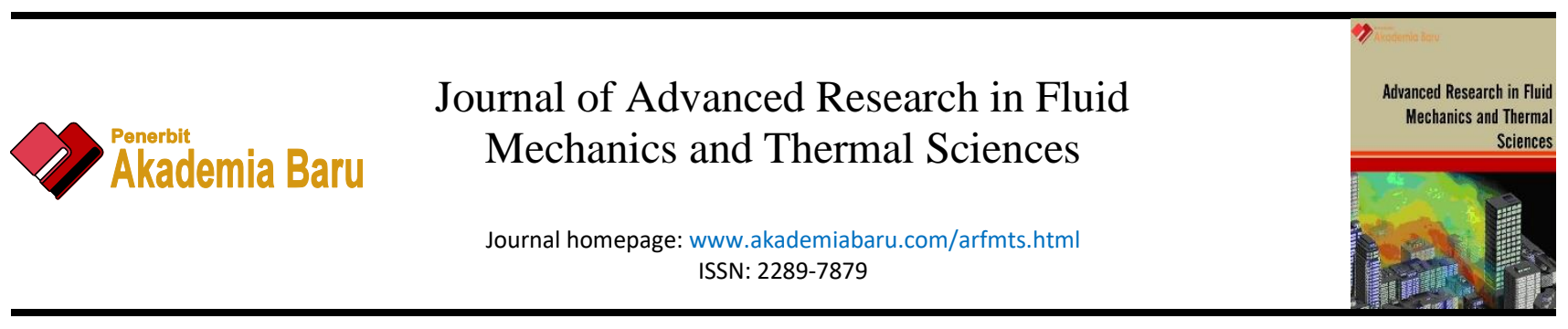

\title{
The Application of Ferric-Metal-Organic Framework for Dye Removal: A Mini Review
}

\author{
Nur Azizah Johari ${ }^{1,2}$, Norhaniza Yusof ${ }^{1,2,{ }^{*}}$, Ahmad Fauzi Ismail ${ }^{1,2}$, Farhana Aziz ${ }^{1,2}$, Wan Norharyati \\ Wan Salleh ${ }^{1,2}$, Juhana Jaafar ${ }^{1,2}$, Nur Hanis Hayati Hairon ${ }^{3}$, Nurasyikin Misdan ${ }^{3}$ \\ 1 Advanced Membrane Technology Research Centre (AMTEC), Universiti Teknologi Malaysia, 81310 Skudai, Johor, Malaysia \\ School of Chemical and Energy, Faculty of Engineering, Universiti Teknologi Malaysia, 81310 Skudai, Johor, Malaysia \\ 3 Faculty of Engineering Technology, Universiti Tun Hussein Onn Malaysia, Hab Pendidikan Tinggi Pagoh, KM 1, Jalan Panchor, 84600, Muar, \\ Johor, Malaysia
}

\section{ARTICLE INFO}

\section{Article history:}

Received 16 February 2020 Received in revised form 6 April 2020 Accepted 7 April 2020

Available online 3 September 2020

\section{Keywords:}

MOF; MIL-100(Fe); Adsorption; Dyes;

\section{ABSTRACT}

\begin{abstract}
Dye effluent released from the industries is one of the most severe environmental concerns due to their potential adverse effects on human health. There are many obstacles encountered in degrading dye materials as they are not only very stable to light and heat but also resistant to biodegradation and oxidation reactions. Currently, porous metal-organic frameworks (MOFs), an important class of advanced functional materials due to its novel coordination structures and diverse topologies, have shown potential applications in various fields, including dye removal. Among the highly water stable MOFs, Ferric-based MOFs like iron (III) trimesate, which is also known as MIL$100(\mathrm{Fe})$, is favorably ascribed by its simple synthesizing method as well as its wide availability. Herein, this paper summarized the recent progress achieved by MIL$100(\mathrm{Fe})$ in removing different types of dye, together with its sustainable preparation method.
\end{abstract}

Copyright $\odot 2020$ PENERBIT AKADEMIA BARU - All rights reserved

\section{Introduction}

Synthetic dyes are valuable in numerous industries, including paper printing, leather, textile, cosmetics, food, and pharmaceutical. However, effluents released from the industries becoming ubiquitous sources of environmental pollution, and their existence has severely affected the aquatic organisms and life cycle. According to statistics, there are currently more than 10,000 types of dyes for commercial purposes, and the yearly output of dyes exceeds 700,000 tons, of which about 10$15 \%$ is released into the environment [1]. With growing concerns regarding a clean environment and human health, technologies with high efficiency and low cost to reduce the pollutant contents of wastewater are urgently needed. To date, various methods have been developed to remove dyes

\footnotetext{
* Corresponding author.

E-mail address: norhaniza@petroleum.utm.my
}

https://doi.org/10.37934/arfmts.75.1.6880 
from aqueous solutions, including biodegradation, photodegradation, and adsorption. The adsorption method is attractive for the removal of dyes because dyes are not only moderately stable to light and heat, but also resistant to oxidation and biodegradation. Besides, the adsorption technique works effectively without the need for additional pretreatment before its application. Though low-cost sorbent material like carbon has been used widely for the adsorption and removal of dyes, the adsorption capacity is quite limited [2]. Thus, it is still a great significance to discover new materials for efficient adsorption for the removal of dyes.

On the other hand, metal-organic-frameworks (MOFs) are a fascinating class of porous crystalline materials self-assembled from metal ions and polyfunctional organic ligands. They have attracted significant research interest in recent years because of their unique characters such as diverse structure topology, tunable pore size, permanent nanoscale porosity, high surface area, good thermostability, and uniform structured cavities [3]. There are growing potential applications of MOFs in recent years, including MOFs intriguing in catalysis [4], gas purification [5,6], selective adsorption [7,8], gas storage [9], and drug delivery [10]. Among known MOFs, ferric-based MOFs like MIL-100(Fe) (MIL stands for Material of Institute Lavoisier) has high-temperature stability, remarkable chemical resistance, unsaturated metal centers with Lewis acid and redox properties, a simple synthesizing method as well as its wide availability of linker and metal sources $[9,10]$, made it the best candidate to be used for wastewater treatment.

Generally, MIL-100(Fe) is generated by coordination self-assembly of Fe (III) octahedral trimmers and trimesic acids (BTC) into hybrid super-tetrahedra (ST), which assemble, giving rise to a rigid micro-mesoporous zeotypic-like structure. It possesses two types of mesoporous cages were $\sim 25 \AA$ and $29 \AA$ in polyhedral shape, in which small cages (SC) accessible through pentagonal windows of $5.5 \AA$ and large cages (LC) also delimited by the bigger hexagonal opening of $8.6 \AA$, as described in Figure 1(a). MIL-100(Fe) has a polyhedron shape (Figure $1(\mathrm{~b})$ ), and an average crystal size less than $1 \mu \mathrm{m}$ with BET specific surface areas and pore volumes ranged from 2500 to $4500 \mathrm{~m}^{2} / \mathrm{g}$ and from 1.2 to $2.0 \mathrm{~cm}^{3} / \mathrm{g}$, respectively [9,11-12].

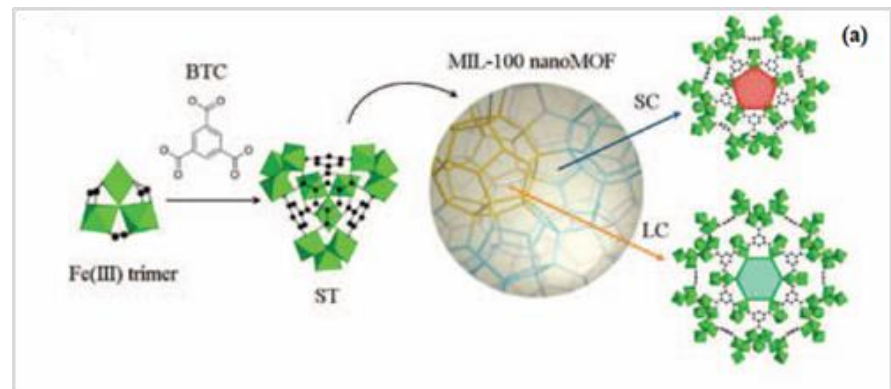

Fig. 1. (a) Illustration of self-assembly coordination and (b) SEM image of MIL-100(Fe) [15]

As MIL-100(Fe) is iron-based metal, it is more suitable for industrial use in comparison with copper, cobalt, or chromium-based materials regarding the toxicity of the metal towards water and microorganism has made it become a quite attractive MOFs material for dye wastewater treatment [14]. Moreover, increasing scientific research of MIL-100(Fe)-based material over the past few years, in which mainly discussing several topics including its unique physicochemical properties, preparation methods, and promising material to be used in the wastewater treatment process, as shown in Figure 2.

A couple of papers have reviewed the MIL-series for different applications in general $[14,15]$. However, there is still no paper mainly reviewed on MIL-100(Fe) for dye removal application. Herein, this mini-review summarized the recent progress achieved by MIL-100(Fe) in removing dyes from aqueous solution, and briefly reviewed the preparation of MIL-100(Fe) on improving its sustainability 
from the conventional process. This paper may provide insights and ideas for researchers who are looking for new hybrid materials, especially in MIL-100(Fe)-based nanostructured, to address the emerging environmental challenge.

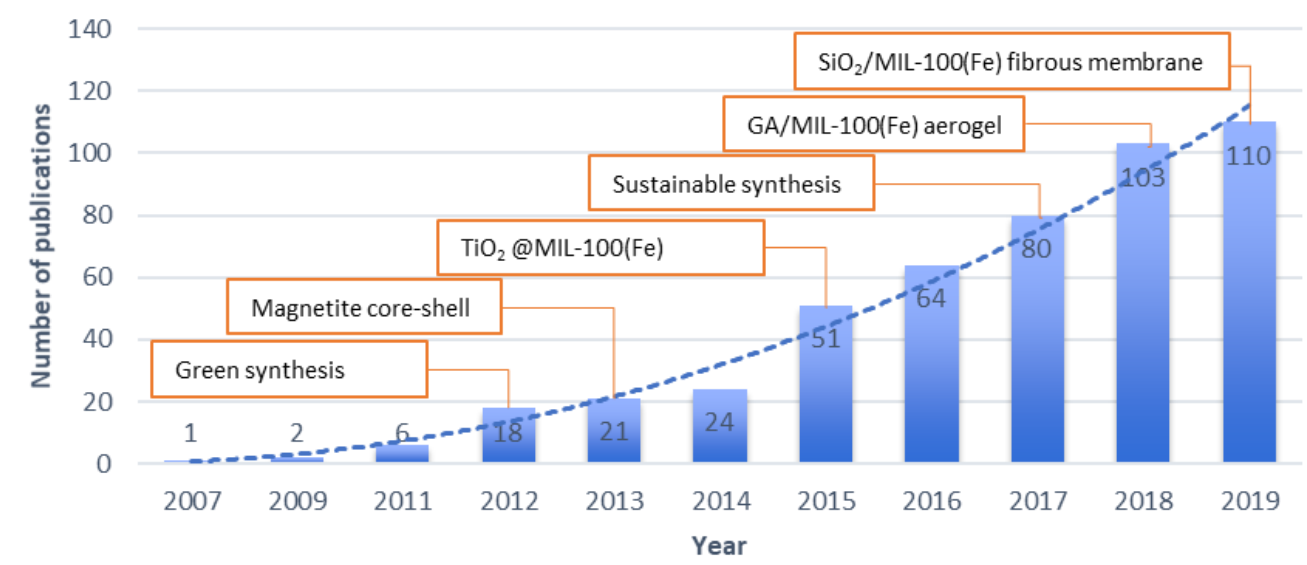

Fig. 2. Growth of research activity of MIL-100(Fe) over the years [Data extracted from the Web of Science database up to December 2019]

\section{Development of Sustainable Preparation of MIL-100(Fe)}

The versatility of MOFs does not only become extraordinarily broad in its physicochemical properties such as porosity, structures, and composition but also a variety of methodologies are available to prepare the material. As such, from fundamental understanding and practical application perspectives, the development of a simple method for MOF synthesis is still a challengeable topic over the past few years.

Horcajada et al., [11] reported the first MIL-100(Fe) preparation method taken from the MIL$100(\mathrm{Cr})$ synthesizing method via hydrothermal treatment with the presence of environmentally harmful and corrosive acids like hydrofluoric acid (HF) and nitric acid $\left(\mathrm{HNO}_{3}\right)$. This method resulting in a very acidic ( $\mathrm{pH}$ less than 1 ) mixture throughout the synthesis, and therefore the recovered solid product required tedious washing steps. Moreover, this hydrothermal method possesses other limitations, such as high synthesis temperature, long synthesis duration, and low product yield in combination with the synthesis conducted at autogenously high pressure.

After that, some research has already shown the efforts to find a green synthesis path with great efficiency by at least avoiding the use of corrosive acids [16-19]. Márquez et al., [18] prepared MIL$100(\mathrm{Fe})$ via a microwave-assisted solvothermal route with green solvents. Despite the effort, it is an unattractive approach due to a low yield of the obtained product makes it difficult for practical use. Besides that, Zhang et al., [20] reported mesoporous MIL-100(Fe) could be synthesized by a simple unappreciated low-temperature $\left(<100^{\circ} \mathrm{C}\right)$ route via the reaction of ferric nitrate and trimesic acid under HF-free conditions. Apart from preparation conditions at room temperature, Sanchez et al., [21] reported semi-amorphous Fe-BTC could be synthesized in the absence of HF, water as the solvent, and a base $\mathrm{NaOH}$ as the deprotonating agent of the linker. However, this method gives the resultant suspension in the acidic range $(\mathrm{pH} 2.1)$, which still required post-treatment to balance the $\mathrm{pH}$. Then, Duan et al., [19] synthesized amorphous MIL-100(Fe) in the absence of $\mathrm{HNO}_{3}$ or $\mathrm{HF}$ at $140^{\circ} \mathrm{C}$ for 12 hours and reported a higher yield of MIL-100(Fe) than the conventional methods. Despite the efforts for green synthesis, these methods still use high operating temperatures or need aggressive post-synthesis washing treatment, thus unfavorable for practical application. 
Recently, Guesh et al., [22] reported a novel synthesis method that modifies from Sanchez et al., [21] and claimed to be the most sustainable and straightforward method for the preparation of highquality MIL-100(Fe) nanoparticles. Although both ways conduct under similar parameters, the only distinction causing the different crystallinity formation was the use of Fe(II) salts instead of Fe(III) as iron sources. As a result, this method gives a mixture of $\mathrm{pH}$ around 5, which is less acidic, thus does not require any aggressive post-synthesis washing treatment at high temperatures-moreover, this simple approach resulting in $76 \%$ of the final product, which is considered practicable.

As a summary for this subtopic, the ideal sustainable preparation of MOFs would include the absence of harmful solvents and corrosive reactants, the use of inexpensive solvents, conducted at room temperature, short synthesis times, non-generation of any harmful by-products, or avoiding long and aggressive post-synthesis washing to purify the product. Further research still needs to be done in synthesizing high-quality MIL-100(Fe) with a sustainable method that gives a higher yield of the product. In short, the development of a cheap, simple, and environmentally friendly approach to synthesis MIL-100(Fe)-based material on a large scale for practical applications is the goal towards worldwide sustainability.

\section{Recent Progress of MIL-100(Fe) on Dyes Removal Application}

Owing to two sets of mesoporous cages that are accessible through microporous windows and corresponding large Langmuir surface area, MIL-100(Fe) has received significant attention concerning its dye adsorption. However, a single adsorption process with MIL-100(Fe) often brings some problems that the contaminants may not be removed entirely because of the adsorption equilibrium [23], and difficult regeneration of MIL-100(Fe) due to their fragile framework [24]. Several modification techniques have used to enhance the properties of MOFs material in water adsorption; (1) modifying the organic ligands with functionalized groups can improve the adsorption behavior of MOFs material towards specified contaminants, (2) altering the pore size and structures of MOFs can attain selective adsorption performance by using different organic linkers or preparation techniques, (3) another modification method is to compound MOFs with other materials to create MOF-based composites that not only limit the drawbacks of MOFs but also achieve synergistic properties. Herein, this paper reviewed the recent progress of MIL-100(Fe) incorporated with other materials, like titanium dioxide $\left(\mathrm{TiO}_{2}\right)$, magnetite $\left(\mathrm{Fe}_{3} \mathrm{O}_{4}\right)$, graphene aerogel, or silica membrane, to form nanocomposites for different applications in achieving a deep removal of dyes in aqueous solution.

\subsection{Photocatalyst}

The organic linkers and transition metal centers in MOFs result in the different ligand-to-metal charge transfer transitions, which make it an efficient photocatalyst [24-27]. Also, MIL-100(Fe) has proved to be a promising compound for photocatalytic performance because $\mathrm{Fe}^{3+}$ and $\mathrm{O}^{2-}$ clusters can be quickly excited by visible irradiation [29]. Unfortunately, low efficient excitons generation and charge separation of MIL-100(Fe) lead to low quantum efficiency in photocatalytic reactions [30]. However, MOFs are commonly known for their highly tunable bandgap due to its huge compositional and structural versatility [21,30-31]. Thus, it could be a plausible strategy to improve the quantum efficiency of MIL-100(Fe) employing the incorporation of MIL-100(Fe) with other materials like semiconductor $\mathrm{TiO}_{2}$, magnetite $\mathrm{Fe}_{3} \mathrm{O}_{4}$, and graphitic carbon nitride $\left(\mathrm{g}-\mathrm{C}_{3} \mathrm{~N}_{4}\right)$. Unmodified MIL-100(Fe) photocatalyst is usually able to degrade around $60 \%$ of $50 \mathrm{mg} / \mathrm{L}$ dyes in aqueous solution at $\mathrm{pH} 5$ under visible light irradiation within $180 \mathrm{~min}$ [33]. In comparison, incorporation with other materials 
improved the removal efficiency up to $100 \%$ in the same conditions within 100 min, as shown in Table 1. Notably, a synergistic effect between MIL-100(Fe) and other nanomaterials enhanced photocatalytic performance, mainly attributed to the high separation efficiency of photo-induced electrons from the lowest unoccupied molecular orbital (LUMO) of other materials injected into the MOF cages of MIL-100(Fe) matrix [34].

Table 1

Recent modification of MIL-100(Fe) as photocatalyst

\begin{tabular}{|c|c|c|c|c|c|c|}
\hline Material & Synthesis method & Operating condition & $\begin{array}{l}\text { Band gap } \\
\text { (eV) }\end{array}$ & Dye & $\begin{array}{l}\% \\
\text { Removal }\end{array}$ & Ref. \\
\hline $\begin{array}{l}\text { Nanosheet } \mathrm{TiO}_{2} / \\
\text { MIL-100(Fe) }\end{array}$ & $\begin{array}{l}\text { Step-by-step self- } \\
\text { assembly method. }\end{array}$ & $\begin{array}{l}\text { Vis light, } \\
150 \text { min }\end{array}$ & 2.52 & $\mathrm{MB}$ & 100 & [35] \\
\hline $\begin{array}{l}\text { N-doped } \mathrm{TiO}_{2} @ M I L- \\
\text { 100(Fe) }\end{array}$ & $\begin{array}{l}\text { Self-assembly chemical } \\
\text { precipitation. }\end{array}$ & $\begin{array}{l}\text { Vis light, } \\
80 \text { min }\end{array}$ & 2.50 & $\begin{array}{l}\mathrm{MB} \\
\mathrm{Rh} B\end{array}$ & $\begin{array}{l}99.1 \\
93.5\end{array}$ & [36] \\
\hline $\begin{array}{l}\text { MIL-100(Fe)/ } \\
\text { Titania Nanofiber }\end{array}$ & $\begin{array}{l}\text { Sustainable, Chemical } \\
\text { precipitation }\end{array}$ & $\begin{array}{l}\text { Vis light, } \\
150 \text { min }\end{array}$ & 2.53 & $\mathrm{MB}$ & 99.4 & [37] \\
\hline MIL-100(Fe) @Bi $2 \mathrm{~S}_{3}$ & Hydrothermal & $\begin{array}{l}\text { Vis light, } \\
60 \mathrm{~min}\end{array}$ & 2.00 & $\mathrm{Rh} B$ & 94 & [38] \\
\hline $\begin{array}{l}\text { Protonated g- } \mathrm{C}_{3} \mathrm{~N}_{4} \\
\text { coated MIL-100(Fe) }\end{array}$ & $\begin{array}{l}\text { In situ protonation and } \\
\text { coating }\end{array}$ & $\begin{array}{l}\text { Vis light, } \\
100 \text { min }\end{array}$ & 2.64 & $\begin{array}{l}\text { MB, } \\
\text { Rh B }\end{array}$ & $\begin{array}{l}100 \\
100\end{array}$ & [39] \\
\hline $\begin{array}{l}\text { Amberlite IRA200 } \\
\text { @FeBTC }\end{array}$ & Chemical precipitation & $\begin{array}{l}\text { Vis light, } \\
60 \mathrm{~min}\end{array}$ & 2.31 & $\mathrm{Rh} B$ & 88.8 & [25] \\
\hline CNNS/MIL-100(Fe) & $\begin{array}{l}\text { Two-step calcination- } \\
\text { exfoliation, solvothermal }\end{array}$ & $\begin{array}{l}\text { Presence of } \mathrm{H}_{2} \mathrm{O}_{2} \text {, } \\
\text { Vis light, } 4 \mathrm{~h}\end{array}$ & 2.88 & $\mathrm{Rh} B$ & 100 & [34] \\
\hline $\mathrm{Fe}_{3} \mathrm{O}_{4} @ \mathrm{MIL}-100(\mathrm{Fe})$ & Solvothermal & $\begin{array}{l}\text { Vis light, } \\
200 \text { min }\end{array}$ & - & $\mathrm{MB}$ & 99.77 & [40] \\
\hline
\end{tabular}

Note: $M B$ is methylene blue; Rh $B$ is Rhodamine $B$

Taking an example of photocatalysis by nanosheet- $\mathrm{TiO}_{2} @ \mathrm{MIL}-100(\mathrm{Fe})$ nanocomposite, the basic mechanism during the photodegradation of dyes over hybrid photocatalyst proposed by Liu and coworkers is illustrated in Figure 3 [35]. The photo-excited electrons ( $\left.\mathrm{e}^{-}\right)$transfer from the valence band to the conduction band on MIL-100(Fe), and holes $\left(\mathrm{h}^{+}\right)$are produced in MIL-100(Fe)'s valence band. In addition, photo-excited electrons ( $\left.\mathrm{e}^{-}\right)$transfer from the conduction band of MIL-100(Fe) to that of nanosheet- $\mathrm{TiO}_{2}$ through the interaction between nanosheet- $\mathrm{TiO}_{2}$ and $\mathrm{MIL}-100(\mathrm{Fe})$, which suppresses the recombination of photogenerated electron-hole pairs and improves the efficiency of photocatalytic activity. The photo-excited holes $\left(\mathrm{h}^{+}\right)$in the valence band of MIL-100(Fe) reacts directly with $\mathrm{H}_{2} \mathrm{O} / \mathrm{OH}^{-}$to generate hydrogen peroxide $(\bullet \mathrm{OH})$. As such, both the photogenerated holes $\left(\mathrm{h}^{+}\right)$and the formed $\bullet \mathrm{OH}$ can directly oxidize the adsorbed organic molecules.

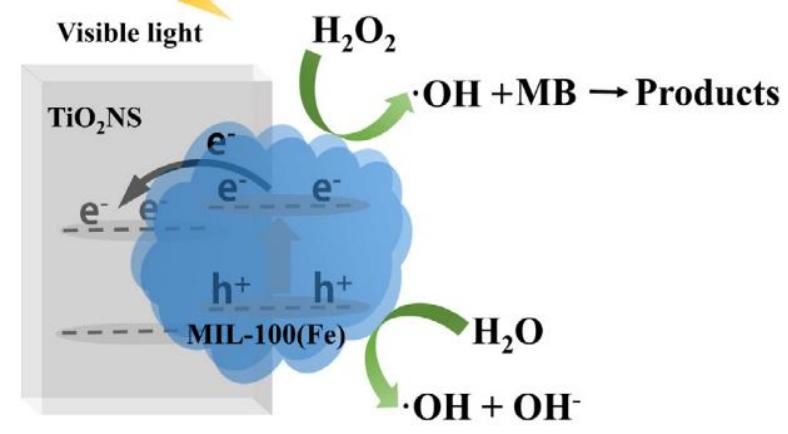

Fig. 3. Photodegradation mechanism of $M B$ by nanosheet-TiO ${ }_{2} @ M I L-100(F e)$ nanocomposite [35] 


\subsection{Adsorbent}

Standalone MIL-100(Fe) adsorbent required tedious and laborious centrifugation both in preparation and applications limits its practical applications. To solve this problem, considerable efforts have been made in the past few years to incorporate $\mathrm{Fe}_{3} \mathrm{O}_{4}$ nanoparticles with MOFs for dye removal due to its properties, including high saturation magnetization, biocompatibility, and low toxicity to microorganisms $[40,41]$. Several published research synthesized $\mathrm{Fe}_{3} \mathrm{O}_{4} @ M I L-100(\mathrm{Fe})$ coreshell adsorbent to remove dye from aqueous solution and proved that the hybrid adsorbent could be easily collected and recycled by a magnetic field due to its super-magnetic characteristic, as shown in Figure 4. These adsorbents achieved maximum adsorption towards dyes around $221 \mathrm{mg} / \mathrm{g}$ up to $625 \mathrm{mg} / \mathrm{g}$ and retained 96\% of removal efficiency after six cycles, in comparison to pristine MIL100(Fe) adsorbent [22,42-44]. Liu et al., [46] studied the adsorption behavior of $\mathrm{Fe}_{3} \mathrm{O}_{4} @ \mathrm{MIL}-100(\mathrm{Fe})$ in terms of adsorption isotherm, kinetics, and thermodynamics. They highlighted that it followed Freundlich isotherm and pseudo-second-order and has a spontaneous endothermic reaction.

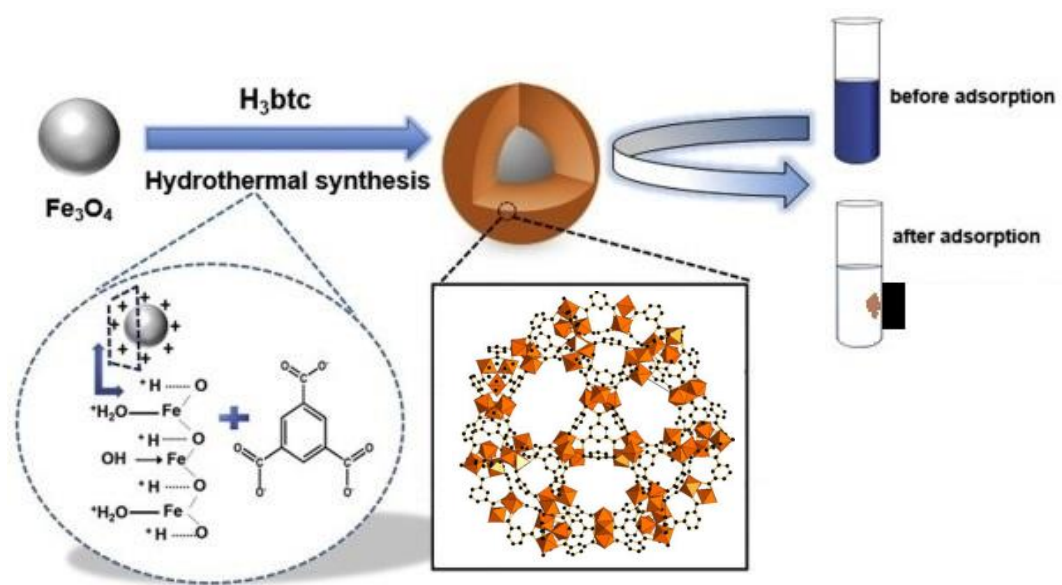

Fig. 4. Schematic diagram of dye removal by $\mathrm{Fe}_{3} \mathrm{O}_{4} @ \mathrm{MIL}-100(\mathrm{Fe})$ core-shell

Additional to that, Fan et al., [47] reported hybrid nanocomposite by immobilization of $\mathrm{Fe}_{3} \mathrm{O}_{4} @ \mathrm{MIL}-100(\mathrm{Fe})$ core-shell on $P$. putida bacteria cells via a carbon-diimide cross-linking method, as illustrated in Figure 5. This hybrid adsorbent degrades dyes completely by following a pseudosecond-order model within $5 \mathrm{~h}$ at an initial dye concentration of $25 \mathrm{mg} / \mathrm{L}$, which is attributed to the synergistic process of adsorption coupled with biodegradation. Apart from it can be easily separated from the solution; this bio-adsorbent may have excellent potential to be applied in real industrial wastewater treatment.

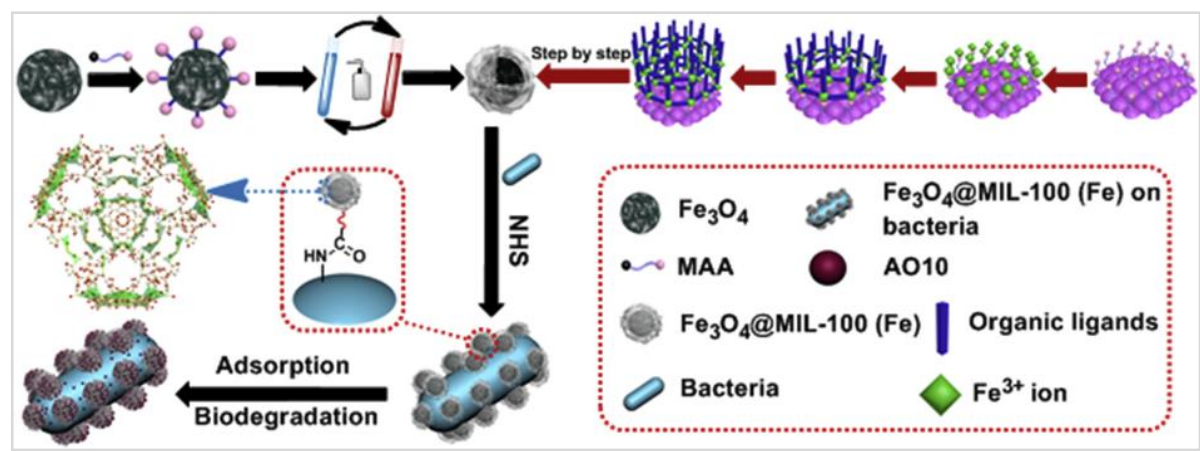

Fig. 5. Schematic diagram of preparation hybrid bio-adsorbent [47] 


\subsection{Aerogel}

Even though MIL-100(Fe)-based photocatalyst or adsorbent could be a promising nanocomposite to degrade dyes by significant researches over the years, however, the powdered crystalline state limits the industrial applicability of MOFs due to the technical challenges of powders such as poor handling and mass transfer limitations $[47,48]$. On the other hand, graphene aerogel inherits the properties of graphene, especially having the structure of a macro-material body, high accessible surface areas, and tunable macropores, and thus have attracted significant attention in adsorption. Thus, hybrid aerogel MIL-100(Fe)/GA seems to be a great potential to solve the issue.

Wan et al., [49] reported an in-situ decoration procedure to load MIL-100(Fe) on the graphene aerogel to form nanocomposite, a three-dimensional hybrid aerogel, where the synergetic effect of catalysis by MIL-100(Fe) and adsorption by graphene aerogel took place, as shown in Figure 6 . Initially, MIL-100(Fe)/GA aerogel adsorbed MB from aqueous solution, then catalyzed the degradation of $\mathrm{MB}$ by the activation of $\mathrm{H}_{2} \mathrm{O}_{2}$. It has achieved maximum adsorption capacity up to $3333.33 \mathrm{mg} / \mathrm{g}$, in comparison with MIL-100(Fe)-based adsorbent, via a pseudo-second-order adsorption process and pseudo-first-order catalytic degradation kinetics. They highlighted that it has the capability to remove highly concentrated pollutants without leaving secondary pollution and retained $93.4 \%$ of its removal efficiency after five consecutive cycles. The excellent regeneration of this macro-material body with a sponge-like shape promotes the stability of hybrid aerogel and contributes to the development of reusable water treatment materials.
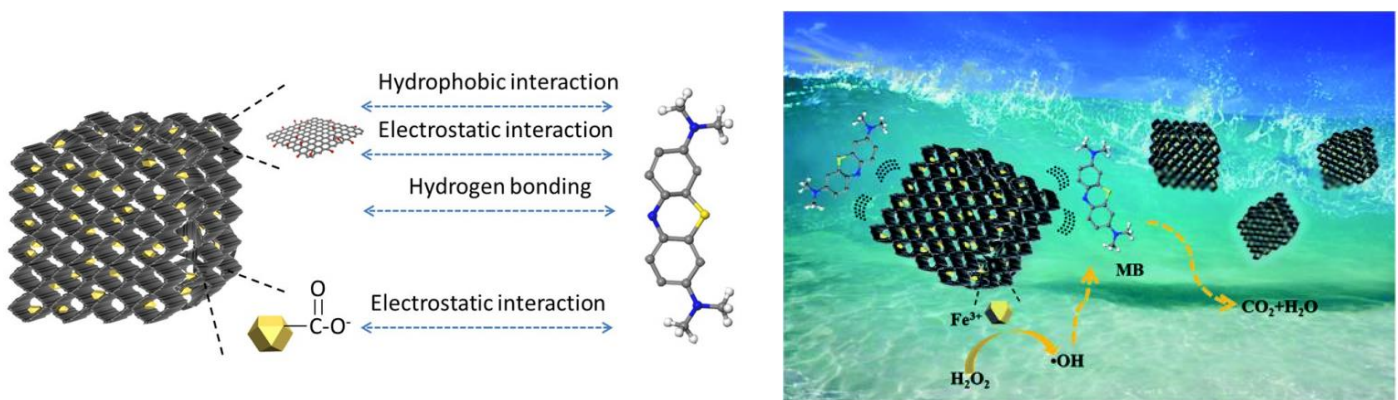

Fig. 6. Illustration of the synergistic adsorption-catalysis coupled process of MB removal by MIL-100(Fe)/GA [49]

\subsection{Hybrid Membrane}

The powder state of the adsorbent or catalyst is not conducive to recycling due to the submicron or micron size of MOF particles, which usually led to a time-consuming and high-cost recycling process. In order to develop more effective MIL-100(Fe)-based materials for dye removal, the construction of MIL-100(Fe) supported fibrous membrane is one of the optional methods. Besides, this hybrid membrane offers not only separation but also adsorption coupled with photocatalytic properties simultaneously, making it the most suitable approach to be applied for treating industrial dye effluent. Chang et al., [50] fabricated a flexible-structured $\mathrm{SiO}_{2} / \mathrm{MIL}-100$ (Fe) fibrous membrane via immobilization of MIL-100(Fe) on the $\mathrm{SiO}_{2}$ fiber surface (Figure 7) for RhB dye degradation under visible light irradiation. This hybrid photocatalytic membrane possesses a 12.6 times larger specific surface area of $79.11 \mathrm{~m}^{2} / \mathrm{g}$ than that of other reported fibrous membranes, which enhanced dye degradation up to $95 \%$ within 70 min irradiation with a reaction rate constant of $0.05 \mathrm{~min}^{-1}$. They claimed the membrane could be washed with deionized water only after each photocatalysis reaction and ready to be used for the next cycle. Besides, the photocatalytic activity remained above $80 \%$ within 90 min illumination after three cycles. These results have proven the reusability and stability 
of $\mathrm{SiO}_{2} / \mathrm{MIL}-100(\mathrm{Fe})$ fibrous membrane than can be potentially applied in larger-scale water treatment.

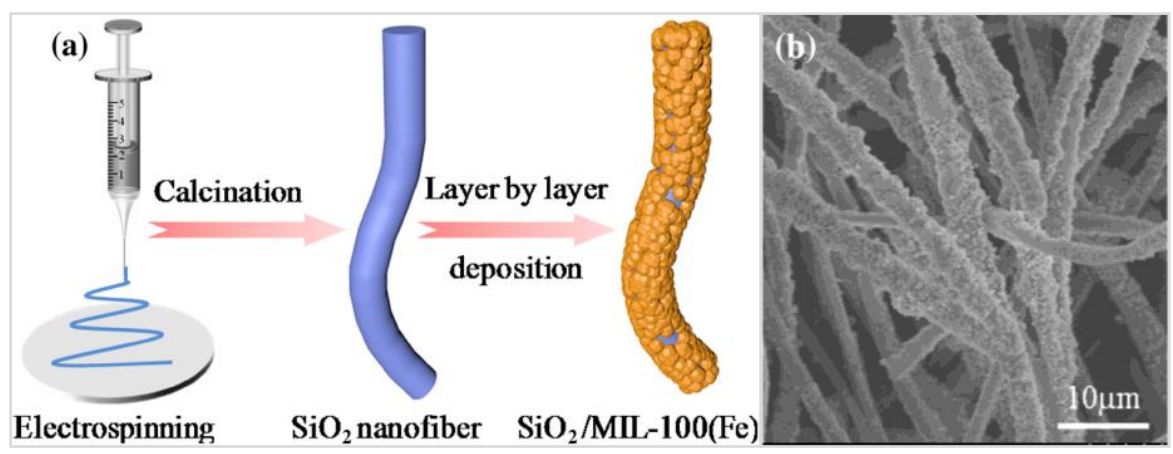

Fig. 7. Fabrication steps (a) and SEM image(b) of $\mathrm{SiO}_{2} / \mathrm{MIL}-100(\mathrm{Fe})$ fibrous membrane [50]

MIL-100(Fe) has excellent capability in dyes removal; however, the hydrophobic nature of MIL100(Fe) limits the development of MIL-100(Fe)-based membrane [51]. Recently, Cho et al., [52] modified MIL-100(Fe) by doping it with chitosan, then employed to PVDF matrix to enhance an antibacterial property, as shown in Figure 8. Apart from improved antibacterial effect, they claimed that the composite of chitosan-MIL-100(Fe) also enhanced hydrophilicity properties of the membrane, thus improving water flux up to $18 \%$, in comparison to pristine PVDF and PVDF/MIL$100(\mathrm{Fe})$ membranes. This may due to deacetylated chitosan has enriched functional groups including hydroxyl $(\mathrm{OH})$ and amino $\left(\mathrm{NH}_{2}\right)$ groups; indirectly, it provides extremely high hydrophilicity to MIL$100(\mathrm{Fe})$ and facilitates a form of bonding with bacteria cells [53]. The excellent dual properties of PVDF/chitosan-MIL-100(Fe) composite membrane enabled the much higher biofouling resistance $\left(\mathrm{J}_{\mathrm{W}} / \mathrm{J}_{\mathrm{W}_{0}}=\mathrm{ca} .0 .85\right)$ compared to PVDF membrane $\left(\mathrm{J}_{\mathrm{W}} / \mathrm{J}_{\mathrm{W}_{0}}=\mathrm{ca} .0 .51\right)$ after $24 \mathrm{~h}$ operation in the crossflow system with the E. coli broth feed solution.
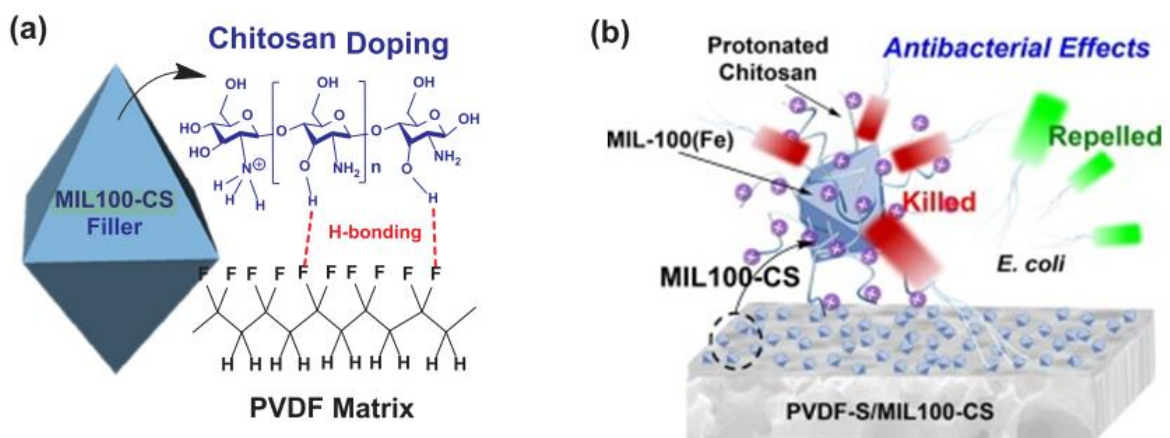

Fig. 8. Schematic diagram of PVDF/MIL-100(Fe)-CS [52]

\section{Limitation and Future Perspective}

The above-mentioned studies indicate that the unique pore structure of MIL-100(Fe) and its incorporation with other materials contribute to the development of water treatment materials. Hybrid adsorbent, aerogel, and membrane solve the problem of that standalone powdered-MIL100 (Fe) photocatalyst and adsorbent, in terms of reusability and recyclability. Among that, the hybrid membrane is a promising material to be applied for industrial wastewater treatment. However, the use of MIL-100(Fe)-based membrane for efficient water treatment is a new and far-reaching area. In addition, from the reviewed literature, the development of MIL-100(Fe)-based membrane lags 
behind despite its aforementioned advantages. This may occur due to the hydrophobic nature of MIL-100(Fe) itself. Thus, improving the hydrophilic properties of MIL-100(Fe) could indirectly enhance the compatibility of $\mathrm{MIL}-100(\mathrm{Fe})$ and nanocomposite membrane for water treatment applications.

Besides that, MIL-100(Fe) shown a positive outcome in dye removal, however, the fabrication methods and testing are still at the laboratory scale, and their readiness for commercialization is in its infancy. Challenges arise when scaling up the technology from lab-scale to industrial-level processes. This can be overcome by testing in real wastewater from industries and employing more realistic conditions to analyses the practicability of MIL-100(Fe)-based materials in treating dye effluents. With continuous research, it is possible to evaluate the effectiveness of MIL-100(Fe)-based materials on a large scale, including long-term stability of the materials under a practical application.

\section{Conclusions}

This paper has reviewed the development of a simple and environmentally friendly method conducted at room temperature with the absence of harmful solvents and corrosive reactants, and a simple washing step to synthesis MIL-100(Fe) or known as a sustainable method. Besides that, this paper reviewed the incorporation of $\mathrm{MIL}-100(\mathrm{Fe})$ with other materials such as $\mathrm{TiO}_{2}, \mathrm{Fe}_{3} \mathrm{O}_{4}, \mathrm{GA}$, or $\mathrm{SiO}_{2}$, to form nanocomposites for different applications in achieving a deep removal of dyes in aqueous solution. Despite having intrinsic properties as a standalone material, incorporating MIL$100(\mathrm{Fe})$-based material with other materials, forming MOFs nanocomposite materials with superior properties, as well improving the limitations possessed by pristine MIL-100(Fe). In conclusion, MIL$100(\mathrm{Fe})$-based material may contribute to the great potential application for water treatment in the future due to its advantages, as mentioned earlier.

\section{Acknowledgement}

The authors gratefully acknowledge the financial supports from the Ministry of Education Malaysia and Universiti Teknologi Malaysia through the Malaysia Research University Network grant (R.J130000.7851.4L865), Higher Institution Centre of Excellence (HICoE) grant (R.J090301.7851.4J428), Award Grant (R.J130000.7351.5M002), and Zamalah scholarship.

\section{References}

[1] Department of Environment Malaysia. Environmental Quality (Industrial Effluent) Regulations 2009. DOE Malaysia, 2010.

[2] De Gisi, Sabino, Giusy Lofrano, Mariangela Grassi, and Michele Notarnicola. "Characteristics and adsorption capacities of low-cost sorbents for wastewater treatment: A review." Sustainable Materials and Technologies 9 (2016): 10-40.

https://doi.org/10.1016/j.susmat.2016.06.002

[3] Fan, Lin, and Xiu-Ping Yan. "Evaluation of isostructural metal-organic frameworks coated capillary columns for the gas chromatographic separation of alkane isomers." Talanta 99 (2012): 944-950. https://doi.org/10.1016/i.talanta.2012.07.063

[4] Dhakshinamoorthy, Amarajothi, Mercedes Alvaro, Young Kyu Hwang, You-Kyong Seo, Avelino Corma, and Hermenegildo Garcia. "Intracrystalline diffusion in Metal Organic Framework during heterogeneous catalysis: Influence of particle size on the activity of MIL-100 (Fe) for oxidation reactions." Dalton Transactions 40, no. 40 (2011): 10719-10724. https://doi.org/10.1039/c1dt10826c

[5] Rose, Marcus, Bertram Böhringer, Marc Jolly, Roland Fischer, and Stefan Kaskel. "MOF processing by electrospinning for functional textiles." Advanced Engineering Materials 13, no. 4 (2011): 356-360. https://doi.org/10.1002/adem.201000246 
[6] Yulia, Fayza, Nasruddin, Agustino Zulys, and Rizky Ruliandini. "Metal-organic framework based chromium terephthalate (MIL-101 Cr) growth for carbon dioxide capture: A review." Journal of Advanced Research in Fluid Mechanics and Thermal Sciences 57, no. 2 (2019): 158-174.

[7] Leclerc, Hervé, Alexandre Vimont, Jean-Claude Lavalley, Marco Daturi, Andrew D. Wiersum, Philip L. Llwellyn, Patricia Horcajada, Gérard Férey, and Christian Serre. "Infrared study of the influence of reducible iron (III) metal sites on the adsorption of $\mathrm{CO}, \mathrm{CO} 2$, propane, propene and propyne in the mesoporous metal-organic framework MIL-100." Physical Chemistry Chemical Physics 13, no. 24 (2011): 11748-11756.

https://doi.org/10.1039/c1cp20502a

[8] Kahlib, Nor Amyra Zulianey, Farah Diana Mohd Daud, Maizirwan Mel, and Ahmad Zahirani Ahmad. "Synthesis and characterization of silica ceramic membranes via sol-dip coating." Journal of Advanced Research in Material Science 39, no. 1 (2017): 1-7.

https://doi.org/10.30967/ijcrset.1.S1.2018.378-383

[9] Lee, Ji Sun, Sung Hwa Jhung, Ji Woong Yoon, Young Kyu Hwang, and Jong-San Chang. "Adsorption of methane on porous metal carboxylates." Journal of Industrial and Engineering Chemistry 15, no. 5 (2009): 674-676. https://doi.org/10.1016/j.jiec.2009.09.043

[10] Cai, Wen, Haiyan Gao, Chengchao Chu, Xiaoyong Wang, Junqing Wang, Pengfei Zhang, Gan Lin, Wengang Li, Gang Liu, and Xiaoyuan Chen. "Engineering phototheranostic nanoscale metal-organic frameworks for multimodal imaging-guided cancer therapy." ACS Applied Materials \& Interfaces 9, no. 3 (2017): 2040-2051.

https://doi.org/10.1021/acsami.6b11579

[11] Horcajada, Patricia, Suzy Surblé, Christian Serre, Do-Young Hong, You-Kyong Seo, Jong-San Chang, Jean-Marc Grenèche, Irene Margiolaki, and Gérard Férey. "Synthesis and catalytic properties of MIL-100 (Fe), an iron (III) carboxylate with large pores." Chemical Communications 27 (2007): 2820-2822.

https://doi.org/10.1039/B704325B

[12] Yoon, Ji Woong, You-Kyong Seo, Young Kyu Hwang, Jong-San Chang, Hervé Leclerc, Stefan Wuttke, Philippe Bazin et al. "Controlled reducibility of a metal-organic framework with coordinatively unsaturated sites for preferential gas sorption." Angewandte Chemie International Edition 49, no. 34 (2010): 5949-5952. https://doi.org/10.1002/anie.201001230

[13] Férey, Gérard. "Hybrid porous solids: past, present, future." Chemical Society Reviews 37, no. 1 (2008): $191-214$. https://doi.org/10.1039/B618320B

[14] Küsgens, Pia, Marcus Rose, Irena Senkovska, Heidrun Fröde, Antje Henschel, Sven Siegle, and Stefan Kaskel. "Characterization of metal-organic frameworks by water adsorption." Microporous and Mesoporous Materials 120, no. 3 (2009): 325-330. https://doi.org/10.1016/j.micromeso.2008.11.020

[15] Lv, Huanli, Hongying Zhao, Tongcheng Cao, Lin Qian, Yanbin Wang, and Guohua Zhao. "Efficient degradation of high concentration azo-dye wastewater by heterogeneous Fenton process with iron-based metal-organic framework." Journal of Molecular Catalysis A: Chemical 400 (2015): 81-89.

https://doi.org/10.1016/i.molcata.2015.02.007

[16] Adeyemo, Aderonke Ajibola, Idowu Olatunbosun Adeoye, and Olugbenga Solomon Bello. "Metal organic frameworks as adsorbents for dye adsorption: overview, prospects and future challenges." Toxicological \& Environmental Chemistry 94, no. 10 (2012): 1846-1863. https://doi.org/10.1080/02772248.2012.744023

[17] Zhong, Guihao, Dingxin Liu, and Jianyong Zhang. "Applications of Porous Metal-Organic Framework MIL-100 (M)(M= Cr, Fe, Sc, Al, V)." Crystal Growth \& Design 18, no. 12 (2018): 7730-7744. https://doi.org/10.1021/acs.cgd.8b01353

[18] Márquez, Alfonso García, Aude Demessence, Ana Eva Platero-Prats, Daniela Heurtaux, Patricia Horcajada, Christian Serre, Jong-San Chang et al. "Green Microwave Synthesis of MIL-100 (Al, Cr, Fe) Nanoparticles for Thin-Film Elaboration." European Journal of Inorganic Chemistry 2012, no. 32 (2012): 5165-5174. https://doi.org/10.1002/ejic.201200710

[19] Duan, Shengxia, Jiaxing Li, Xia Liu, Yanan Wang, Suyuan Zeng, Dadong Shao, and Tasawar Hayat. "HF-free synthesis of nanoscale metal-organic framework NMIL-100 (Fe) as an efficient dye adsorbent." ACS Sustainable Chemistry \& Engineering 4, no. 6 (2016): 3368-3378.

https://doi.org/10.1021/acssuschemeng.6b00434

[20] Zhang, Fumin, Jing Shi, Yan Jin, Yanghe Fu, Yijun Zhong, and Weidong Zhu. "Facile synthesis of MIL-100 (Fe) under HF-free conditions and its application in the acetalization of aldehydes with diols." Chemical Engineering Journal 259 (2015): 183-190. https://doi.org/10.1016/i.cej.2014.07.119 
[21] Sanchez-Sanchez, Manuel, Iñigo de Asua, Daniel Ruano, and Kenya Diaz. "Direct synthesis, structural features, and enhanced catalytic activity of the basolite F300-like semiamorphous Fe-BTC framework." Crystal Growth \& Design 15, no. 9 (2015): 4498-4506.

https://doi.org/10.1021/acs.cgd.5b00755

[22] Guesh, Kiros, Clarice AD Caiuby, Álvaro Mayoral, Manuel Díaz-García, Isabel Díaz, and Manuel Sanchez-Sanchez. "Sustainable preparation of MIL-100 (Fe) and its photocatalytic behavior in the degradation of methyl orange in water." Crystal Growth \& Design 17, no. 4 (2017): 1806-1813.

https://doi.org/10.1021/acs.cgd.6b01776

[23] Tong, Minman, Dahuan Liu, Qingyuan Yang, Sabine Devautour-Vinot, Guillaume Maurin, and Chongli Zhong. "Influence of framework metal ions on the dye capture behavior of MIL-100 (Fe, Cr) MOF type solids." Journal of Materials Chemistry A 1, no. 30 (2013): 8534-8537. https://doi.org/10.1039/c3ta11807i

[24] Tsai, Fang-Chang, Yue Xia, Ning Ma, Jing-Jing Shi, Tao Jiang, Tai-Chin Chiang, Zuo-Cai Zhang, and Wen-Chin Tsen. "Adsorptive removal of acid orange 7 from aqueous solution with metal-organic framework material, iron (III) trimesate." Desalination and Water Treatment 57, no. 7 (2016): 3218-3226. https://doi.org/10.1080/19443994.2014.982199

[25] Araya, Tirusew, Chun-cheng Chen, Man-ke Jia, David Johnson, Ruiping Li, and Ying-ping Huang. "Selective degradation of organic dyes by a resin modified Fe-based metal-organic framework under visible light irradiation." Optical Materials 64 (2017): 512-523. https://doi.org/10.1016/j.optmat.2016.11.047

[26] Mosleh, S., M. R. Rahimi, M. Ghaedi, K. Dashtian, S. Hajati, and Shaobin Wang. "Ag3PO4/AgBr/Ag-HKUST-1-MOF composites as novel blue LED light active photocatalyst for enhanced degradation of ternary mixture of dyes in a rotating packed bed reactor." Chemical Engineering and Processing: Process Intensification 114 (2017): 24-38. https://doi.org/10.1016/i.cep.2017.01.009

[27] Thakare, Sanjay R., and Shruti M. Ramteke. "Postmodification of MOF-5 using secondary complex formation using 8-hydroxyquinoline (HOQ) for the development of visible light active photocatalysts." Journal of Physics and Chemistry of Solids 116 (2018): 264-272. https://doi.org/10.1016/i.jpcs.2018.01.032

[28] Chen, Ying, Boyin Zhai, and Yuning Liang. "Enhanced degradation performance of organic dyes removal by semiconductor/MOF/graphene oxide composites under visible light irradiation." Diamond and Related Materials 98 (2019): 107508. https://doi.org/10.1016/i.diamond.2019.107508

[29] Xu, Bo, Zhiming Chen, Bing Han, and Cuncheng Li. "Glycol assisted synthesis of MIL-100 (Fe) nanospheres for photocatalytic oxidation of benzene to phenol." Catalysis Communications 98 (2017): 112-115. https://doi.org/10.1016/i.catcom.2017.04.041

[30] Ke, Fei, Luhuan Wang, and Junfa Zhu. "Facile fabrication of CdS-metal-organic framework nanocomposites with enhanced visible-light photocatalytic activity for organic transformation." Nano Research 8, no. 6 (2015): 18341846. https://doi.org/10.1007/s12274-014-0690-x

[31] Fan, Jixiang, Dongyun Chen, Najun Li, Qingfeng Xu, Hua Li, Jinghui He, and Jianmei Lu. "Adsorption and biodegradation of dye in wastewater with Fe304@ MIL-100 (Fe) core-shell bio-nanocomposites." Chemosphere 191 (2018): 315-323.

https://doi.org/10.1016/i.chemosphere.2017.10.042

[32] Mohammadifard, Zahra, Rahmatallah Saboori, Naghmeh Sadat Mirbagheri, and Samad Sabbaghi. "Heterogeneous photo-Fenton degradation of formaldehyde using MIL-100 (Fe) under visible light irradiation." Environmental Pollution 251 (2019): 783-791. https://doi.org/10.1016/i.envpol.2019.04.143

[33] Mahmoodi, Niyaz Mohammad, Jafar Abdi, Mina Oveisi, Mokhtar Alinia Asli, and Manouchehr Vossoughi. "Metalorganic framework (MIL-100 (Fe)): Synthesis, detailed photocatalytic dye degradation ability in colored textile wastewater and recycling." Materials Research Bulletin 100 (2018): 357-366.

https://doi.org/10.1016/j.materresbull.2017.12.033

[34] Hong, Jindui, Chunping Chen, Franky E. Bedoya, Geoff H. Kelsall, Dermot O'Hare, and Camille Petit. "Carbon nitride nanosheet/metal-organic framework nanocomposites with synergistic photocatalytic activities." Catalysis Science \& Technology 6, no. 13 (2016): 5042-5051. https://doi.org/10.1039/C5CY01857A 
[35] Liu, Xin, Rui Dang, Wenjun Dong, Xiubing Huang, Jia Tang, Hongyi Gao, and Ge Wang. "A sandwich-like heterostructure of TiO2 nanosheets with MIL-100 (Fe): a platform for efficient visible-light-driven photocatalysis." Applied Catalysis B: Environmental 209 (2017): 506-513.

https://doi.org/10.1016/i.apcatb.2017.02.073

[36] Huang, Jie, Haiyan Song, Chunxia Chen, Yi Yang, Ningdi Xu, Xinzhen Ji, Chunyu Li, and Jiang-An You. "Facile synthesis of N-doped TiO2 nanoparticles caged in MIL-100 (Fe) for photocatalytic degradation of organic dyes under visible light irradiation." Journal of Environmental Chemical Engineering 5, no. 3 (2017): 2579-2585.

https://doi.org/10.1016/i.jece.2017.05.012

[37] Oveisi, Mina, Niyaz Mohammad Mahmoodi, and Mokhtar Alinia Asli. "Facile and green synthesis of metal-organic framework/inorganic nanofiber using electrospinning for recyclable visible-light photocatalysis." Journal of Cleaner Production 222 (2019): 669-684.

https://doi.org/10.1016/i.jclepro.2019.03.066

[38] Abdpour, Soheil, Elaheh Kowsari, Mohammad Reza Alavi Moghaddam, Laura Schmolke, and Christoph Janiak. "Mil100 (Fe) nanoparticles supported on urchin like Bi2S3 structure for improving photocatalytic degradation of rhodamine-B dye under visible light irradiation." Journal of Solid State Chemistry 266 (2018): 54-62.

https://doi.org/10.1016/i.jssc.2018.07.006

[39] Huang, Jie, Xibiao Zhang, Haiyan Song, Chunxia Chen, Fuqin Han, and Congcong Wen. "Protonated graphitic carbon nitride coated metal-organic frameworks with enhanced visible-light photocatalytic activity for contaminants degradation." Applied Surface Science 441 (2018): 85-98.

https://doi.org/10.1016/j.apsusc.2018.02.027

[40] Zhang, Chao-Feng, Ling-Guang Qiu, Fei Ke, Yu-Jun Zhu, Yu-Peng Yuan, Geng-Sheng Xu, and Xia Jiang. "A novel magnetic recyclable photocatalyst based on a core-shell metal-organic framework Fe304@ MIL-100 (Fe) for the decolorization of methylene blue dye." Journal of Materials Chemistry A 1, no. 45 (2013): 14329-14334. https://doi.org/10.1039/c3ta13030d

[41] Li, Ling, Li Jun Yuan, Wei Hong, Lu Fan, Le Bao Mao, and Li Liu. "Hybrid Fe304/MOFs for the adsorption of methylene blue and methyl violet from aqueous solution." Desalination and Water Treatment 55, no. 7 (2015): 1973-1980. https://doi.org/10.1080/19443994.2014.937751

[42] Liu, Jia, Zhenkun Sun, Yonghui Deng, Ying Zou, Chunyuan Li, Xiaohui Guo, Liqin Xiong, Yuan Gao, Fuyou Li, and Dongyuan Zhao. "Highly water-dispersible biocompatible magnetite particles with low cytotoxicity stabilized by citrate groups." Angewandte Chemie International Edition 48, no. 32 (2009): 5875-5879.

https://doi.org/10.1002/anie.200901566

[43] Dadfarnia, S., AM Haji Shabani, S. E. Moradi, and S. Emami. "Methyl red removal from water by iron based metalorganic frameworks loaded onto iron oxide nanoparticle adsorbent." Applied Surface Science 330 (2015): 85-93. https://doi.org/10.1016/i.apsusc.2014.12.196

[44] Aslam, Sobia, Jingbin Zeng, Fazle Subhan, Min Li, Fenglei Lyu, Yanpeng Li, and Zifeng Yan. "In situ one-step synthesis of Fe304@ MIL-100 (Fe) core-shells for adsorption of methylene blue from water." Journal of Colloid and Interface Science 505 (2017): 186-195.

https://doi.org/10.1016/j.jcis.2017.05.090

[45] Huo, Shu-Hui, and Xiu-Ping Yan. "Metal-organic framework MIL-100 (Fe) for the adsorption of malachite green from aqueous solution." Journal of Materials Chemistry 22, no. 15 (2012): 7449-7455. https://doi.org/10.1039/c2jm16513a

[46] Liu, Haochi, Xiaohui Ren, and Ligang Chen. "Synthesis and characterization of magnetic metal-organic framework for the adsorptive removal of Rhodamine B from aqueous solution." Journal of Industrial and Engineering Chemistry 34 (2016): 278-285.

https://doi.org/10.1016/i.jiec.2015.11.020

[47] Fan, Jixiang, Dongyun Chen, Najun Li, Qingfeng Xu, Hua Li, Jinghui He, and Jianmei Lu. "Adsorption and biodegradation of dye in wastewater with Fe304@ MIL-100 (Fe) core-shell bio-nanocomposites." Chemosphere 191 (2018): 315-323.

https://doi.org/10.1016/i.chemosphere.2017.10.042

[48] Dong, Ruifang, Dongyun Chen, Najun Li, Qingfeng Xu, Hua Li, Jinghui He, and Jianmei Lu. "Enhancement of organic pollutants bio-decontamination from aqueous solution using newly-designed Pseudomonas putida-GA/MIL-100 (Fe) bio-nanocomposites." Environmental Research 173 (2019): 237-245. https://doi.org/10.1016/i.envres.2019.03.052

[49] Wan, Yinjia, Jianzhi Wang, Fei Huang, Yanan Xue, Ning Cai, Jie Liu, Weimin Chen, and Faquan Yu. "Synergistic effect of adsorption coupled with catalysis based on graphene-supported MOF hybrid aerogel for promoted removal of dyes." Royal Society of Chemistry Advances 8, no. 60 (2018): 34552-34559.

https://doi.org/10.1039/C8RA05873C 
[50] Chang, Meng-Jie, Wen-Na Cui, Xiao-Jiao Chai, Jun Liu, Kang Wang, and Lei Qiu. "Fabrication of flexible MIL-100 (Fe) supported SiO 2 nanofibrous membrane for visible light photocatalysis." Journal of Materials Science: Materials in Electronics 30, no. 2 (2019): 1009-1016.

https://doi.org/10.1007/s10854-018-0370-9

[51] Yang, Shuliang, Li Peng, Daniel T. Sun, Mehrdad Asgari, Emad Oveisi, Olga Trukhina, Safak Bulut, Abbas Jamali, and Wendy L. Queen. "A new post-synthetic polymerization strategy makes metal-organic frameworks more stable." Chemical Science 10, no. 17 (2019): 4542-4549.

https://doi.org/10.1039/C9SC00135B

[52] Cho, Kie Yong, Cheol Hun Yoo, Young-June Won, Jong-San Chang, Jae-Woo Choi, Jung-Hyun Lee, and Jong Suk Lee. "Surface-concentrated chitosan-doped MIL-100 (Fe) nanofiller-containing PVDF composites for enhanced antibacterial activity." European Polymer Journal 120 (2019): 109221.

https://doi.org/10.1016/i.eurpolymj.2019.109221

[53] Ong, Chi Siang, P. S. Goh, W. J. Lau, Nurasyikin Misdan, and A. Fauzi Ismail. "Nanomaterials for biofouling and scaling mitigation of thin film composite membrane: A review." Desalination 393 (2016): 2-15.

https://doi.org/10.1016/j.desal.2016.01.007 